\title{
BMJ Open Patient-reported safety incidents in older patients with long-term conditions: a large cross-sectional study
}

\author{
Maria Panagioti, ${ }^{1}$ Thomas Blakeman, ${ }^{1,2}$ Mark Hann, ${ }^{1}$ Peter Bower ${ }^{1,3}$
}

To cite: Panagioti $M$, Blakeman T, Hann $\mathrm{M}$, et al. Patient-reported safety incidents in older patients with long-term conditions: a large crosssectional study. BMJ Open 2017;7:e013524. doi:10.1136/ bmjopen-2016-013524

- Prepublication history and additional material are available. To view these files please visit the journal online (http://dx.doi. org/10.1136/bmjopen-2016013524).

Received 19 July 2016 Revised 26 December 2016 Accepted 3 January 2017

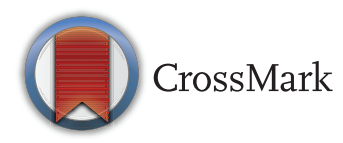

${ }^{1}$ NIHR School for Primary Care Research Centre for Primary Care, Manchester Academic Health Science Centre, University of Manchester, Manchester, UK

${ }^{2}$ National Institute for Health Research Collaboration for Leadership in Applied Health Research and Care (NIHR CLAHRC) Greater Manchester, Centre for Primary Care, University of Manchester, Manchester, UK

${ }^{3}$ NIHR Greater Manchester Primary Care Patient Safety Translational Research Centre (Greater Manchester PSTRC), Manchester Academic Health Science Centre University of Manchester, Manchester, UK

Correspondence to Dr Maria Panagioti; maria.panagioti@manchester. ac.uk

\section{ABSTRACT}

Background Increasing evidence suggests that patient safety is a serious concern for older patients with longterm conditions. Despite this, there is a lack of research on safety incidents encountered by this patient group. In this study, we sought to examine patient reports of safety incidents and factors associated with reports of safety incidents in older patients with long-term conditions. Methods The baseline cross-sectional data from a longitudinal cohort study were analysed. Older patients $(n=3378$ aged 65 years and over) with a long-term condition registered in general practices were included in the study. The main outcome was patient-reported safety incidents including availability and appropriateness of medical tests and prescription of wrong types or doses of medication. Binary univariate and multivariate logistic regression analyses were undertaken to examine factors associated with patient-reported safety incidents.

Results Safety incidents were reported by $11 \%$ of the patients. Four factors were significantly associated with patient-reported safety incidents in multivariate analyses. The experience of multiple long-term conditions $(\mathrm{OR}=1.09$, $95 \% \mathrm{Cl} 1.05$ to 1.13 ), a probable diagnosis of depression $(\mathrm{OR}=1.36,95 \% \mathrm{Cl} 1.06$ to 1.74$)$ and greater relational continuity of care $(\mathrm{OR}=1.28,95 \% \mathrm{Cl} 1.08$ to 1.52$)$ were associated with increased odds for patient-reported safety incidents. Perceived greater support and involvement in self-management was associated with lower odds for patient-reported safety incidents $(\mathrm{OR}=0.95,95 \% \mathrm{Cl} 0.93$ to 0.97 ).

Conclusions We found that older patients with multimorbidity and depression are more likely to report experiences of patient safety incidents. Improving perceived support and involvement of patients in their care may help prevent patient-reported safety incidents.

\section{INTRODUCTION}

Patient safety is defined as the 'avoidance, prevention, and amelioration of adverse outcomes or injuries stemming from the processes of healthcare', while a patient safety incident is defined as 'any unintended event or hazardous condition resulting from the process of care, rather than due to the patient's underlying disease, that led or could have led to unintended health consequences for the patient or healthcare processes linked to safety outcomes'. ${ }^{2}$ Patient safety research
Strengths and limitations of this study

- This is the first large quantitative study examining experiences of patient safety incidents in older patients with long-term conditions.

- The study used a cross-sectional design and self-report questionnaires to assess the patients' experiences of safety incidents such as the availability and appropriateness of medical tests and the prescription of wrong types or doses of medication.

- Experiences of safety incidents were reported by around 1 in 10 older patients with long-term conditions. Multimorbidity and a probable diagnosis of depression were the key factors associated with experiencing patient safety incidents. Perceived enhanced involvement and support in selfmanagement was associated with a lower likelihood of experiencing patient safety incidents.

- This work was undertaken in one region using a brief measure of patient safety and therefore may have limitations in terms of completeness of patient safety experiences and representation of the full range of older patients with long-term conditions in the UK.

has traditionally focused on hospital-based specialist settings, but there is growing evidence that patient safety in primary care can also be problematic. ${ }^{3-5}$ Patient safety incidents related to prescription of medication, diagnostic errors and communication failures are frequently encountered in primary and secondary care (ranging from $2 \%$ to $10 \%$ of consultations) ${ }^{6-9}$

These safety incidents might be more common or severe in vulnerable patient groups such as older patients with two or more long-term conditions (known as multimorbidity). ${ }^{10}{ }^{11}$ Multimorbidity is one of the key challenges facing healthcare systems and a key determinant of patient experience, health outcomes, mortality and costs. ${ }^{1112}$ A recent systematic review has shown that patients with multimorbidity are potentially vulnerable to patient safety incidents, 
which may include active adverse (drug or other) events, prescription errors, medication non-adherence and diagnostic errors. However, evidence about prevalence and the mechanisms driving safety incidents in patients with multimorbidity is limited. ${ }^{10}$ There are several reasons that might explain why patients with multimorbidity are more likely to experience patient safety incidents. The experience of multiple long-term conditions combined with the patients' poor health, ${ }^{13}$ high rates of depression, ${ }^{14}$ increased age ${ }^{215}$ and low levels of health literacy ${ }^{16} 17$ are likely to magnify the patients' perception of 'treatment burden' associated with the management of complex management regimes ${ }^{18}$ and to reduce their ability to reach difficult decisions between treatments and courses of action. ${ }^{19}$ Patients with multimorbidity may have to deal with multiple professionals in different care contexts, making them vulnerable to problems in coordination. ${ }^{20}$ Furthermore, the understanding of safety in primary care patients incorporates psychosocial and moral dimensions. ${ }^{21}$ Patients highlight the importance of establishing an ongoing and trusting relationship with their healthcare provider for reducing the possibility of psychological harm and safety incidents. ${ }^{22}$

There is a developing literature on the aspects of the healthcare system that are drivers of quality in the care of long-term conditions, largely based on the Chronic Care Model (CCM). ${ }^{23}$ Although these aspects do not directly refer to patient safety, the causes of failures in quality of care and safety often overlap and therefore similar approaches might be effective in sustaining high quality and safety of care. Care coordination, defined as 'the deliberate organization of patient care activities between two or more participants (including the patient) involved in a patient's care to facilitate the appropriate delivery of health care services', ${ }^{24}$ is likely to be an important determinant of safety for people with multimorbidity. Patients with multimorbidity often receive care from multiple professionals in different contexts. ${ }^{25}$ Continuity of care has several aspects (relational, informational and management), ${ }^{26}$ some of which are related to coordinating functions, but research has highlighted the role of relational continuity, an ongoing therapeutic relationship between a patient and one or more providers, ${ }^{26}$ in enhancing patients' sense of safety. ${ }^{22}$ Moreover, patient involvement in their own care and self-management is seen as critical for quality of care in long-term conditions, through the provision of information and care plans, support for shared decision making and higher levels of patient participation in care. ${ }^{27}$ Active patients engaged in self-management may contribute to safety through better communication and identification of developing safety issues. $^{28} 29$

Although there has been extensive qualitative work around the patient experience of multimorbidity burden, ${ }^{12}{ }^{30}$ there has been less assessment of the patient experience of safety, especially using quantitative methods. This is an important omission as evidence suggests that patient reports reliably recognise a range of problems in healthcare delivery ${ }^{31}$ and have potential to improve the systematic detection of problems in healthcare that are not identified by traditional systems. ${ }^{32}{ }^{33} \mathrm{In}$ this study, we used quantitative data from a large community sample of older patients with long-term conditions to assess the patient-reported experience of safety incidents. The objectives of the study were:

1. to describe the frequency of patient-reported safety incidents patients with long-term conditions;

2. to identify key characteristics of patients with longterm conditions and their perceptions of healthcare delivery system that are associated with patientreported safety incidents. Based on existing evidence, we hypothesised that patient characteristics such as multimorbidity, depression and lower levels of health literacy would be associated with heightened rates of patient-reported safety incidents. Aspects of quality of the healthcare system (such as continuity of care, co-coordination of care and enhanced involvement and support of patients in self-management) were expected to be associated with reduced rates of patient-reported safety incidents.

\section{METHODS}

\section{Participants and recruitment}

This study describes a cross-sectional analysis of the baseline data of a large longitudinal cohort study-the Comprehensive Longitudinal Assessment of Salford Integrated Care (CLASSIC). The completed STROBE checklist is included as supplementary file. Participants in CLASSIC had to meet two main inclusion criteria: (1) aged 65 years or over and (2) being registered as having at least one long-term condition at a general practice in Salford, North West of England. Individuals receiving palliative care, and those with a reduced capacity to consent and participate, were excluded from this study.

Salford has a population of 294916 (34000 aged over 65 years) and total of 52 general practices (clustered in eight neighbourhoods) and 33 practices $(65 \%)$ agreed to take part. A list of potentially eligible participants in each participating general practice was created using the FARSITE software (a tool for recruitment to research studies in primary care). Afterwards, we liaised with each practice to identify any patients who meet the exclusion criteria of the study. No incentives were offered to practices, but support costs were provided as a reimbursement of their time.

A total of 12989 patients were finally eligible for participation. Questionnaires were sent to participants by post between November 2014 and February 2015. Those who did not return the first questionnaire were sent a reminder letter and a second copy of the questionnaire 3 weeks later. Participants were reimbursed with a $£ 10$ voucher. 


\section{Dependent variable}

Patient-reported safety incidents was assessed using four items from the integrated care pilot evaluation. ${ }^{34}$ Two of these items focus on availability and appropriateness of medical tests in the past 6 months: 'Test results on your medical notes were not available at the time of your appointment' and 'The doctor or nurse ordered a test that you felt was unnecessary because it had already been done' and two items on prescribing wrong types of doses of medication in the past 6 months: 'You were given the wrong medicine or drug' and 'You were given the wrong dose of a medicine or drug'. These items were rated with responses of 'Yes', 'No' or 'Don't know'. For the purposes of the analyses, 'Yes' responses were coded as 1, whereas 'No' or 'Don't know' responses were coded as 0 .

\section{Independent variables}

Sociodemographic characteristics

Age, gender, ethnicity and qualifications were assessed using questions derived by the General Practice Patient Survey. ${ }^{35}$

Health literacy was assessed using the Single Item Literacy Screener (rated from $1=$ never to $5=$ always) ${ }^{36}$ : 'How often do you need to have someone help you when you read instructions, pamphlets, or other written material from your doctor in pharmacy?' This measure has been previously used in people with long-term conditions and demonstrated good reliability and validity. ${ }^{3637}$

\section{Long-term conditions}

A validated questionnaire was used to assess the self-reported number and burden of long-term conditions. ${ }^{38}$ This questionnaire contains 21 common long-term conditions and also allows patients to report additional conditions not already on the list. Participants can rate each condition on a five-point scale that assesses interference with the daily activities ( $1=$ not at all to $5=\mathrm{a}$ lot $)$. The total burden score is the sum of conditions weighted by the level of interference assigned to each. ${ }^{38}$

\section{Depression}

The presence of depression was assessed using the Mental Health Inventory (MHI-5), a five-item scale, which incorporates questions on general mental health, including depression, anxiety, behavioural-emotional control and general positive affect in the past month. ${ }^{39}$ This measure is well validated for identifying depression symptoms with a higher score indicating better mental health. ${ }^{40}{ }^{41}$ The recommended cut-off score of 60 was used to indicate the presence of 'probable depression'.

\section{Patient-centred and coordinated care}

The short version of the Patient Assessment of Care for Chronic Conditions (PACIC-S) ${ }^{42}$ self-report measure was used. The PACIC-S contains 11 items (rated from 1= almost never to $5=$ almost always) and is a patient-centred assessment of implementation of the CCM, which focuses on the receipt of patient-centred care, coordination and self-management behaviour over the last 6 months. A higher score indicates higher self-reported patient-centred care and coordination. PACIC-S has satisfactory psychometric properties including validity, reliability and sensitivity to change. ${ }^{42}$

Patient involvement and support to self-management

The Long Term Conditions 6 (LTC-6) questionnaire was used to assess perceptions of involvement and support in self-management. It consists of six questions (responses range from $1=$ almost never to $5=$ almost always) that ask patients with a long-term condition about their healthcare over the last 12 months. ${ }^{43}$ Its focus in on perceived support to self-management and also includes questions about involvement in decision making. A higher score indicates more involvement and receipt of better support in self-management.

\section{Relational continuity of care}

Relational continuity was assessed using two items focused on preference for and success in seeing a preferred doctor: 'Is there one doctor you prefer to see at your GP surgery or health centre?' and 'How often do you see the doctor you prefer to see at your GP surgery or health centre?' These two items were derived from the 2009/2010 English General Practitioner Patient Survey, ${ }^{35}$ which is a national questionnaire survey covering different aspects of patient experience of English primary care practice.

\section{Data analysis}

Descriptive statistics of the variables in the analyses including means and SDs for continuous variables, and counts and percentages for categorical variables were computed.

For all the analyses, one binary variable for patient-reported safety incidents was constructed, which categorised patients as to whether they had reported one or more of any of the four safety issues, or none at all. This approach was deemed necessary as some types of safety incidents were rarely reported by participants (ranged from $3.1 \%$ to $5.9 \%$ of the sample). Furthermore, since very few patients reported more than one safety incidents (less than $1 \%$ of the sample), we decided that treating patient-reported safety incidents as an ordinal/continuous outcome would not be a sensible approach. Only cases with valid scores on patient safety incidents were included in the analyses. However, imputations were performed for independent variables with missing values. In line with recommendations, linear, binary logistic and multinomial logistic imputations were generated for each independent variable with missing values using the other independent variables as predictors. Following the multiple imputation process, 602 cases were added in the analyses. As a validation exercise, the analyses were performed with and without the imputed cases; no differences were observed in the results.

Univariate logistic regression analyses were performed to examine the association between each explanatory variable and patient-reported safety incidents (dependent 
variable). Multivariate logistic regression analysis was next applied using all variables from the univariate analyses with $p$ values $\leq 0.1$ (to avoid prematurely excluding important associations) as predictors of patient-reported safety incidents. A backwards elimination procedure was used to sequentially remove predictors with the highest $p$ values until all remaining variables were significant at the $\mathrm{p}<0.05$ level.

The variance inflation factors (VIFs) values were inspected to assess the levels of multicollinearity between explanatory variables. The McKelvey and Zavoina $\mathrm{R}^{2}$, which estimates explained variance in a latent continuous variable underlying observed awareness (yes/no), was used to estimate the overall predictive power of the logistic regression. This measure was used to ease interpretation as it is comparable with explained variance in linear regression. ${ }^{44}$ All analyses were conducted using Stata V.14.

\section{RESULTS}

The descriptive characteristics of participants and the key variables in the analyses are summarised in table 1. A total of 4377 out of $12989(33.6 \%)$ individuals returned the questionnaire at baseline. A percentage of $51.2(n=1726)$ of participants were women, and the vast majority were white $(98.5 \% ; \mathrm{n}=3309)$. The mean age of participants was 74.5 years $(\mathrm{SD}=6.8)$, and they reported a mean number of $5.6(\mathrm{SD}=3.1)$ long-term conditions.

$3378(77.2 \%)$ of the 4377 participants provided complete data for the analyses. Approximately $11 \%$ of the participants $(n=367)$ reported at least one type of safety incident (see table 2). From those 367 participants, $8.5 \%(n=291)$ reported experiences of unavailability or inappropriateness of test results, whereas $3.3 \% \quad(n=102)$ reported that they have been prescribed a wrong type or dose of medication. Only a very small minority of patients $(n=31 ; 0.9 \%)$ reported more than one safety incident.

Factors associated with the patient-reported safety incidents The results of the univariate logistic regression analysis are presented in table 1. Patients who reported safety incidents were significantly more likely to live alone, have lower levels of health literacy, greater number of long-term conditions and have 'probable depression'. Patient-reported safety incidents were less likely among patients who were satisfied with the support they receive for self-management and felt more involved in the decisions relating to their self-management (as measured by LTC-6). Higher relational continuity of care measured by patient-reported availability and access to a preferred doctor was associated with increased odds for safety incidents. Aspects of the CCM (including patient-centredness and coordination of care), age and gender were not significantly associated with safety incidents $(\mathrm{p}>0.05)$.

VIFs among the explanatory variables entered into the multivariate analysis were all low (maximum=1.8), indicating acceptable multicollinearity ${ }^{29}$. Four factors remained significant predictors of patient-reported safety incidents (see table 3). A higher number of long-term conditions ( $\mathrm{OR}=1.09,95 \%$ CI 1.05 to 1.13$)$, "probable depression' ( $\mathrm{OR}=1.36,95 \%$ CI 1.06 to 1.74 ) and greater relational continuity ( $\mathrm{OR}=1.28,95 \%$ CI 1.08 to 1.52$)$ were associated with increased odds for patient-reported safety incidents. Perceived higher support and involvement in self-management were associated with lower odds for patient-reported safety incidents ( $\mathrm{OR}=0.95,95 \%$ CI 0.03 to 0.97 ). The model as a whole explained approximately $6 \%$ of the variance in patient-reported safety incidents (McKelvey and Zavoina's $\mathrm{R}^{2}=0.057$ ).

\section{DISCUSSION}

This is the first empirical study that examined factors associated with patient-reported safety incidents encountered by a community sample of older patients with long-term conditions. Approximately 1 in 10 patients with long-term conditions reported a patient safety incident over the past 6 months. Having a greater number of long-term conditions and a diagnosis of depression were the two key patient characteristics associated with an increased likelihood of patient-reported safety incidents. Perceived strong support and involvement in the self-management was inversely associated with safety incidents. An unexpected finding was that greater relational continuity (assessed by the perceived availability and access to a preferred doctor) was associated with an increased probability for reporting of safety incidents.

\section{Strengths and limitations}

The main strength of this study is that it examined an area that has received sparse research attention despite the potential serious effects of patient safety incidents on the health of vulnerable patients. Other major strengths of this study comprise the large sample size, the focus on older patients who are the most frequent utilisers of primary health services but are often excluded by research studies and the use of a patient-centred perspective in evaluating the presence and associates of patient safety incidents.

However, there are also important limitations. First, the cross-sectional design of this study does not allow any casual inferences to be drawn for the association between patient-reported safety incidents and predictors included in the regression models. Prospective designs are necessary to establish causal relationships.

Second, approximately one-third of all eligible patients $(34 \%)$ agreed to take part in this study. Our response rate is consistent with the response rates of other studies using similar methods and populations. ${ }^{45}$ However, non-response bias might operate, and therefore we suggest caution in interpreting these findings. Particular caution should be exercised with regards to the accuracy of our estimates of the prevalence of patient-reported safety incidents. 


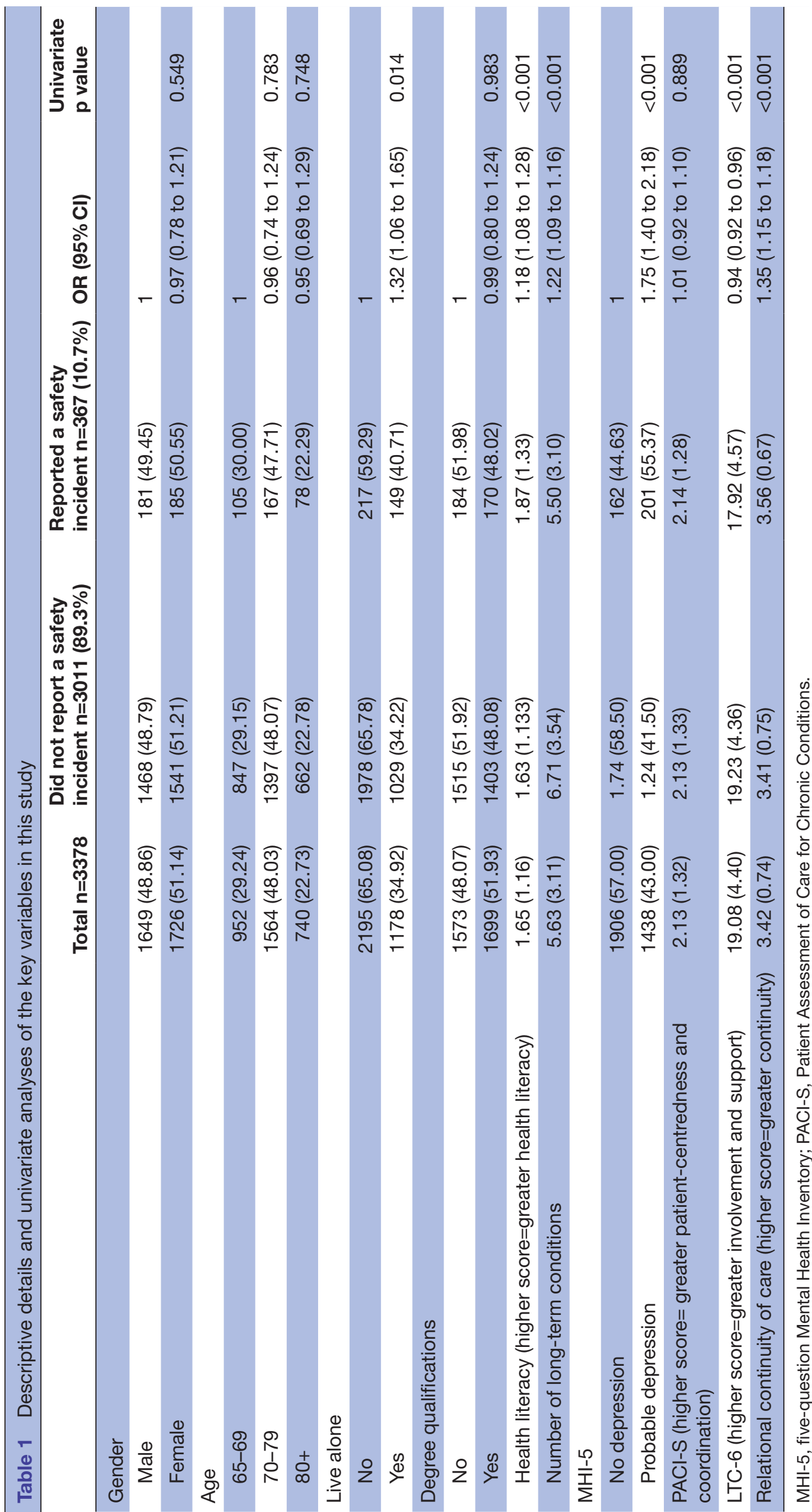




$\begin{aligned} & \text { Table } 2 \\
& \text { patients with long-term conditions }\end{aligned}$
\begin{tabular}{ll} 
Types of self-reported safety incidents & N (\%) \\
\hline Unavailability of the results of medical tests & $182(5.18)$ \\
\hline Ordering unnecessary medical tests & $109(3.50)$ \\
Given wrong type of medication & $56(1.80)$ \\
Given wrong dose of medication & $46(1.48)$
\end{tabular}

A third limitation is that a brief assessment of patient safety incidents was undertaken using four items that enquired participants to self-report safety incidents related to medical tests and prescription of medication. Inappropriate prescription of medication and medical tests were highlighted as two key sources of safety failures for people with multimorbidity. However, other types of safety incidents such as diagnostic errors and communication failures were also found to be important threats to safety but were not measured in this study. ${ }^{6-9}$ Furthermore, the small proportion of patients reporting safety incidents in this study meant that examining predictors of each safety incident separately and predictors of counts of safety incidents were not possible. Thus, we recognise that assessment of safety incidents and its associated factors in this study is very likely to be incomplete, but no other comprehensive and validated tools for patient-reported experiences of safety were available when this study commenced. A comprehensive measure of patient-reported experiences of safety has been recently published, and we recommended its use by future studies. ${ }^{46}$

We mainly described patient-reported safety incidents as a primary care problem in this study because data were derived from a community sample of patients registered in general practices. However, older people with multimorbidity are high utilisers of healthcare services in general and it is likely that some of the reported incidents to have

Table 3 Multivariate analysis of factors associated with patient safety incidents

\begin{tabular}{lll}
\hline Variable & OR $(95 \%$ Cl) & $\begin{array}{l}\text { Univariate } \\
\text { p value }\end{array}$ \\
\hline $\begin{array}{l}\text { Number of long-term } \\
\text { conditions }\end{array}$ & $1.09(1.05$ to 1.13$)$ & $\mathrm{p}<0.001$ \\
$\begin{array}{l}\text { MHI-5 } \\
\quad \text { Probable depression }\end{array}$ & $1.36(1.06$ to 1.74$)$ & $\mathrm{p}=0.016$ \\
$\quad$ No depression & 1 & \\
$\begin{array}{l}\text { LTC-6 (higher } \\
\text { score=greater } \\
\text { involvement and } \\
\text { support) }\end{array}$ & $0.95(0.03$ to 0.97$)$ & $\mathrm{p}<0.001$ \\
$\begin{array}{l}\text { Continuity of care } \\
\text { (higher score=greater } \\
\text { continuity) }\end{array}$ & $1.28(1.08$ to 1.52$)$ & $\mathrm{p}<0.001$ \\
\hline
\end{tabular}

MHI-5, five-question Mental Health Inventory. occurred in secondary care or in the interface of primary and secondary care (eg, emergency department).

Fifth, the MHI-5 questionnaire used to assess the presence of a probable diagnosis of depression has not been specifically validated for use in primary care settings. Despite this, MHI-5 is a valid measure of depression in general population, has been previously used in people with long-term conditions and is increasingly translated and used in different countries. ${ }^{47}$

Finally, our findings represent the experiences of patients with long-term conditions in one area in the UK primarily composed of white patients. There is evidence that ethnic minority groups report poor experience of care and they might also report more negative experiences of safety. ${ }^{48}$

\section{Meaning of the findings in the context of existing literature}

Our findings suggest that a small but considerable proportion of older people with long-term conditions report patient safety incidents in their interactions with healthcare services. This estimate should be interpreted cautiously for a number of reasons. Comparison with previous literature is not possible because this is the first study that examined the prevalence and nature of safety incidents experienced by older patients with long-term conditions. Our focus was on patients with complex needs and high burden who might report patient safety experiences resulting from different stages of the healthcare delivery process including primary and secondary care. There is also some evidence that patients report experiences of safety incidents more often compared with data contained in healthcare records. ${ }^{49}$

In terms of contributory factors to patient safety incidents, our findings are in agreement with a recent systematic review that showed that severe multimorbidity and comorbid depression increase the risk for patient safety incidents in primary care.$^{10}$ However, it is equally true that patients with depression may report more negatively on their experiences of care,${ }^{50}$ so it will be important for further research to explore whether the effect of depression reflects reporting bias or actual incidents.

Other patient factors such as older age or health literacy levels were not related to patient-reported safety incidents in the multivariate analyses. The only component of the healthcare delivery associated with lower patient-reported safety incidents was a perception of higher support and more active involvement in self-management. This finding is in line with existing data demonstrating the beneficial effects of patient involvement on various processes and outcomes of care ${ }^{27}$ and in healthcare decisions for matching care to patient preferences and for improving the safety and quality of care. ${ }^{28} 29$

Contrary to our expectations, core quality indicators derived by the CCM such as patient-centred care and organisation of care were not associated with lower patient-reporting of safety incidents. This finding could mean that quality of care and safety do not lie on the same continuum or that more effort is needed to translate 
metrics of the CCM into measureable patient-experienced benefits such as high quality and safety of care.

Importantly, greater relational continuity was significantly associated with higher rates of patient-reported safety incidents. Patients with experiences of safety incidents in this study were the frailest subgroup in terms of severity of multimorbidity and depression, also known to be heavy utilisers of all healthcare services including primary and secondary care ${ }^{51}$ Consequently, the link between relational continuity of care and patient-reported safety incidents might be mediated by increased healthcare utilisation rates. ${ }^{52}$ Patients in frequent contact with healthcare services are more likely to experience system failures such as unavailability of test results (the most commonly reported safety problem in our sample) in other healthcare contexts such as hospitals despite their close relationships with their general practitioners (GPs). It is possible that these patients seek out a close relationship with their GP in response to past safety incidents that occurred in secondary care systems, or that a close relationship makes patients more comfortable reporting problems in the past. However, this finding might suggest that familiarity between the patient and doctor is not necessarily an advantage but can also be a source of harm. For example, doctors might fail to perform regular medical assessments because they think they know the history and the needs of the patient. ${ }^{53}$ Moreover, over-reliance and trust in doctor might make patients less vigilant to procedures that might lead to safety incidents (eg, patients might not check prescriptions and recommendations given by a trusted doctor) or might reflect patient's hesitation in raising safety issues during consultations because they do not want to risk this relationship. ${ }^{545}$ The relationship between continuity of care and patient safety in people with multimorbidity warrants further research. We only established a cross-sectional association in this study; an examination of the relationship between continuity and safety over time might reveal a different pattern of findings.

\section{Policy implications and research directions}

From a clinical and policy perspective, these findings confirm that patient safety is a reasonably prevalent problem among patients with long-term conditions and has identified some factors that are associated with higher rates. However, our analyses also confirmed that safety incidents are difficult to predict; our regression model only explained $6 \%$ of the variance in the occurrence of patient-reported safety incidents. A likely explanation is that patient safety incidents often result from a complex and dynamic interplay between patients, doctors (eg, health and workload) and the healthcare system (eg, organisational culture), which is difficult to model.

It is important to be cautious in drawing strong recommendations from this preliminary data. However, our findings support the conclusions of the recent WHO report that puts forward a number of practical steps to improve patient safety for people with multimorbidity such as enhancing communication systems, prioritising research into safely managing people with multimorbidity, identifying people in need for extra support (eg, mental health problems), increasing patient involvement in safety planning and encouraging systemic approaches for improving patient safety in healthcare systems. ${ }^{56}$

More methodologically rigorous studies such as longitudinal cohort studies or longitudinal qualitative studies are needed to confirm the key factors leading to safety incidents over time. A number of studies are currently ongoing, which are expected to improve the understanding of patient safety in the UK primary care. ${ }^{757} 58$ Additionally, the use of a newly validated measure, which is designed to capture patient-reported safety incidents, ${ }^{46}$ could advance the knowledge of the nature, precursors and outcomes of patient-reported safety incidents. Finally, studies incorporating different perspectives of patient safety (accounts of patients, healthcare professionals and health records) are strongly recommended to gain a more complete understanding of patient safety in primary care and generate efficient interventions at the patient and healthcare system level.

Funding MP and PB authored and are guarantors of this manuscript. MP led all aspects of data collection and analysis, and drafted and finalised the manuscript. $\mathrm{TB}$ and $\mathrm{MH}$ assisted with data analysis and critically reviewed the manuscript. $\mathrm{PB}$ conceived the research design, critically revised the manuscript and assisted with data analysis and interpretation. All authors approved the final manuscript. NIHRSchool for Primary Care Research funded some of the time and facilities of MP.

Funding This publication/paper is independent research part funded by the NIHR Greater Manchester Primary Care Patient Safety Translational Research Centre and the National Institute of Health Research Health Services and Delivery Programme (project 12/130/33). The views expressed are those of the author(s) and not necessarily those of the NHS, the NIHR or the Department of Health.

\section{Competing interests None declared.}

Ethics approval This study was approved by the NRES Committee North West Lancaster (Ref: 14/NW/0206), and all participants provided informed consent prior to participation.

Provenance and peer review Not commissioned; externally peer reviewed. Data sharing statement No additional data are available.

Open Access This is an Open Access article distributed in accordance with the Creative Commons Attribution Non Commercial (CC BY-NC 4.0) license, which permits others to distribute, remix, adapt, build upon this work non-commercially, and license their derivative works on different terms, provided the original work is properly cited and the use is non-commercial. See: http://creativecommons.org/ licenses/by-nc/4.0/

(C) Article author(s) (or their employer(s) unless otherwise stated in the text of the article) 2017. All rights reserved. No commercial use is permitted unless otherwise expressly granted.

\section{REFERENCES}

1. Vincent C. Patient safety. Edinburgh: Churchill Livingstone, 2005.

2. Klemp K. Report on a taxonomy for errors in primary care. Wolfgang Goethe University, 2009.

3. Daker-White G, Hays R, McSharry J, et al. Blame the patient, blame the doctor or blame the system? a meta-synthesis of qualitative studies of patient safety in primary care. PLoS One 2015;10:e0128329.

4. Samra R, Bottle A, Aylin P. Monitoring patient safety in primary care: an exploratory study using in-depth semistructured interviews. BMJ Open 2015;5:e008128.

5. Cooper A, Chuter A. Patient safety research in primary care: where are we now? Br J Gen Pract 2015;65:622-3. 
6. Balla J, Heneghan C, Goyder C, et al. Identifying early warning signs for diagnostic errors in primary care: a qualitative study. BMJ Open 2012;2:e001539.

7. Carson-Stevens A, Hibbert P, Avery A, et al. A cross-sectional mixed methods study protocol to generate learning from patient safety incidents reported from general practice. BMJ Open 2015;5:e009079.

8. Klemp K, Dovey S, Valderas JM, et al. Developing a patient safety incident classification system for primary care. A literature review and Delphi-survey by the LINNEAUS collaboration on patient safety in primary care. Eur J Gen Pract 2015;21 Suppl:35-8.

9. Sandars J, Esmail A. The frequency and nature of medical error in primary care: understanding the diversity across studies. Fam Pract 2003;20:231-6.

10. Panagioti M, Stokes J, Esmail A, et al. Multimorbidity and patient safety incidents in primary care: a systematic review and MetaAnalysis. PLoS One 2015;10:e0135947.

11. Bower P. Better management of multimorbidity: a critical look at the 'Ariadne principles'. BMC Med 2014;12:1-3.

12. Coventry PA, Small N, Panagioti M, et al. Living with complexity; marshalling resources: a systematic review and qualitative metasynthesis of lived experience of mental and physical multimorbidity. BMC Fam Pract 2015;16:171.

13. Bayliss EA, Steiner JF, Fernald DH, et al. Descriptions of barriers to self-care by persons with comorbid chronic diseases. Ann Fam Med 2003;1:15-21.

14. Detweiler-Bedell JB, Friedman MA, Leventhal H, et al. Integrating co-morbid depression and chronic physical disease management: identifying and resolving failures in self-regulation. Clin Psychol Rev 2008;28:1426-46.

15. Vogeli C, Shields AE, Lee TA, et al. Multiple chronic conditions: prevalence, health consequences, and implications for quality, care management, and costs. J Gen Intern Med 2007;22 Suppl 3:391-5.

16. van Dijk PT, Mehr DR, Ooms ME, et al. Comorbidity and 1-year mortality risks in nursing home residents. J Am Geriatr Soc 2005;53:660-5.

17. Nutbeam D. The evolving concept of health literacy. Soc Sci Med 2008;67:2072-8.

18. Fried TR, McGraw S, Agostini JV, et al. Views of older persons with multiple morbidities on competing outcomes and clinical decisionmaking. J Am Geriatr Soc 2008;56:1839-44.

19. Bayliss EA, Bosworth HB, Noel PH, et al. Supporting selfmanagement for patients with complex medical needs: recommendations of a working group. Chronic IIIn 2007;3:167-75.

20. Pham HH, O'Malley AS, Bach PB, et al. Primary care physicians' links to other physicians through Medicare patients: the scope of care coordination. Ann Intern Med 2009;150:236-42.

21. Rhodes P, McDonald R, Campbell S, et al. Sensemaking and the co-production of safety: a qualitative study of primary medical care patients. Sociol Health IIIn 2016;38:270-85.

22. Rhodes P, Campbell S, Sanders C, Trust SC. Trust, temporality and systems: how do patients understand patient safety in primary care? A qualitative study. Health Expect 2016;19:253-63.

23. Bodenheimer T, Wagner EH, Grumbach K. Improving primary care for patients with chronic illness: the chronic care model, part 2. JAMA 2002;288:1909-14.

24. McDonald K, Sundaram V. et alClosing the quality gap: a critical analysis of quality improvement strategies (Technical Review 9). In: Shojania K, McDonald K, Wachter R, Owens D, . eds. Care coordinat. Rockville, MD, 2007.

25. Doessing A, Burau V. Care coordination of multimorbidity: a scoping study. J Comorbid 2015;5:15-28.

26. Haggerty JL, Reid RJ, Freeman GK, et al. Continuity of care: a multidisciplinary review. BMJ 2003;327:1219-21.

27. Barry MJ, Edgman-Levitan S. Shared decision making-pinnacle of patient-centered care. N Engl J Med 2012;366:780-1.

28. McDonald KM, Bryce CL, Graber ML. The patient is in: patient involvement strategies for diagnostic error mitigation. BMJ Qual Saf 2013;22 Suppl 2:ii33-ii39.

29. McDonald KM, Matesic B, Contopoulos-loannidis DG, et al. Patient safety strategies targeted at diagnostic errors: a systematic review. Ann Intern Med 2013;158:381.

30. Kenning C, Coventry PA, Gibbons C, et al. Does patient experience of multimorbidity predict self-management and health outcomes in a prospective study in primary care? Fam Pract 2015;32:311-6.

31. Schwappach DL. Review: engaging patients as vigilant partners in safety: a systematic review. Med Care Res Rev 2010;67:119-48.

32. Bjertnaes O, Deilkås ET, Skudal KE, et al. The association between patient-reported incidents in hospitals and estimated rates of patient harm. Int J Qual Health Care 2015;27:26-30.
33. Reader TW, Gillespie A, Roberts J. Patient complaints in healthcare systems: a systematic review and coding taxonomy. BMJ Qual Saf 2014;23:678-89.

34. Scottish patient safety programme in primary care. http://www. scottishpatientsafetyprogramme.scot.nhs.uk/programmes/primarycare

35. Campbell J, Smith P, Nissen S, et al. The GP patient survey for use in primary care in the National Health Service in the UK--development and psychometric characteristics. BMC Fam Pract 2009;10:57.

36. Morris NS, MacLean CD, Chew LD, et al. The single item literacy screener: evaluation of a brief instrument to identify limited reading ability. BMC Fam Pract 2006;7:21.

37. Jeppesen KM, Coyle JD, Miser WF. Screening questions to predict limited health literacy: a cross-sectional study of patients with diabetes mellitus. Ann Fam Med 2009;7:24-31.

38. Bayliss EA, Ellis JL, Steiner JF. Seniors' self-reported multimorbidity captured biopsychosocial factors not incorporated into two other data-based morbidity measures. J Clin Epidemiol 2009;62:550-7.

39. Berwick DM, Murphy JM, Goldman PA, et al. Performance of a fiveitem mental health screening test. Med Care 1991;29:169-76.

40. Yamazaki S, Fukuhara S, Green J. Usefulness of five-item and threeitem Mental Health inventories to screen for depressive symptoms in the general population of Japan. Health Qual Life Outcomes 2005;3:48.

41. Kelly MJ, Dunstan FD, Lloyd K, et al. Evaluating cutpoints for the MHI-5 and MCS using the GHQ-12: a comparison of five different methods. BMC Psychiatry 2008;8:10.

42. Cramm JM, Nieboer AP. Factorial validation of the patient Assessment of chronic illness care (PACIC) and PACIC short version (PACIC-S) among cardiovascular disease patients in the Netherlands. Health Qual Life Outcomes 2012;10:104.

43. NHS, Report of findings from the pilot programme2011 http://www. diabetes.nhs.uk/document.php?o=2926.

44. Veall MR, Zimmermann KF. Pseudo-r 2 measures for some common limited dependent variable models. J Econ Surv 1996;10:241-59.

45. Guthrie EA, Dickens C, Blakemore A, et al. Depression predicts future emergency hospital admissions in primary care patients with chronic physical illness. J Psychosom Res 2016;82:54-61.

46. Ricci-Cabello I, Avery AJ, Reeves D, et al. Measuring patient safety in primary care: the development and validation of the "patient reported experiences and outcomes of safety in primary care" (PREOS-PC). Ann Fam Med 2016;14:253-61.

47. Harrison M, Reeves D, Harkness E, et al. A secondary analysis of the moderating effects of depression and multimorbidity on the effectiveness of a chronic disease self-management programme. Patient Educ Couns 2012;87:67-73.

48. Burt J, Lloyd C, Campbell J, et al. Variations in GP-patient communication by ethnicity, age, and gender: evidence from a national primary care patient survey. $\mathrm{Br} J$ Gen Pract 2016;66:e47-e52.

49. Ricci-Cabello I, Violán C, Foguet-Boreu Q, et al. Impact of multimorbidity on quality of healthcare and its implications for health policy, research and clinical practice. A scoping review. Eur J Gen Pract 2015;21:192-202.

50. Ludvigsson M, Milberg A, Marcusson J, et al. Normal aging or depression? A qualitative study on the differences between subsyndromal depression and depression in very old people. Gerontologist 2015;55:760-9.

51. Dickens C, Katon W, Blakemore A, et al. Does depression predict the use of urgent and unscheduled care by people with long term conditions? A systematic review with meta-analysis. J Psychosom Res 2012;73:334-42.

52. Hobbs FDR, Bankhead C, Mukhtar T, et al. Clinical workload in UK primary care: a retrospective analysis of 100 million consultations in England, 2007-14. The Lancet 2016;387:2323-30.

53. O'Connor E, Coates HM, Yardley IE, et al. Disclosure of patient safety incidents: a comprehensive review. Int J Qual Health Care 2010;22:371-9.

54. Hernan AL, Walker C, Fuller J, et al. Patients' and carers' perceptions of safety in rural general practice. Med J Aust 2014;201:60-3.

55. Hrisos S, Thomson R. Seeing it from both sides: do approaches to involving patients in improving their safety risk damaging the trust between patients and healthcare professionals? an interview study. PLoS One 2013;8:e80759.

56. Mercer S, Fischbacher-Smith D, Furler J, et al; Safer primary care: caring for people with multiple conditions. Geneva: World Health Organisation, 2016.

57. Cheraghi-Sohi S, Singh H, Reeves D, et al. Missed diagnostic opportunities and english general practice: a study to determine their incidence, confounding and contributing factors and potential 
impact on patients through retrospective review of electronic medical records. Implement Sci 2015;10:105.

58. Daker-White G, Hays R, Esmail A, et al. MAXimising involvement in MUltiMorbidity (MAXIMUM) in primary care: protocol for an observation and interview study of patients, GPs and other care providers to identify ways of reducing patient safety failures. BMJ Open 2014;4:e005493. 